\title{
The relationship between cardiopulmonary exercise testing (CPET), other related factors and outcomes in patients undergoing major upper gastrointestinal surgery
}

\author{
Dr. Janan Alrefaee ${ }^{a}$, Professor Donald C McMillan ${ }^{b}$, Dr. Roger Carter ${ }^{c}$, Dr. Jane E. Nally ${ }^{d}$

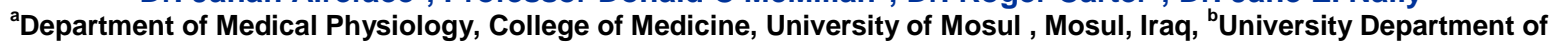 \\ Surgery, Glasgow Royal Infirmary, Glasgow, 'Department of Respiratory Medicine, Glasgow Royal Infirmary, Glasgow, \\ ${ }^{d}$ School of Health and Life Sciences, Department of Life Sciences, Glasgow Caledonian University, Glasgow. \\ Correspondence: Janan Alrefaee. janan1969@yahoo.com.
}

(Ann Coll Med Mosul 2019; 41 (1):1-10).

Received: $4^{\text {th }}$ Oct. 2018; Accepted: $13^{\text {th }}$ Jan. 2019.

\begin{abstract}
Background: Assessment of patient's fitness prior to major surgery is mandatory because co-morbidities predispose to postoperative complications; however the ability of pulmonary function tests to predict postoperative cardiopulmonary complications is still poorly defined.

Objective: to examine the role of preoperative measurements of cardiopulmonary exercise testing and other lung function tests in predicting postoperative outcomes in patients undergoing major upper gastrointestinal surgery (stomach, oesophagus, small intestine, gall bladder and pancreas surgery).

Patients and methods: A 193 patients who had major upper gastrointestinal surgery' age, sex and smoking status were recorded and they underwent a range of tests including body mass index, flow-volume curve spirometry, blood gas analysis, transfer factor for carbon monoxide (TLCO) and CPET at the Pulmonary Function Laboratory of Glasgow Royal Infirmary between August 2008 and December 2011. Post-operative morbidity and mortality was analysed for all patients who underwent major surgery.

Results: Comparisons between patients that did or did not have post operative pulmonary complications showed significant differences in anaerobic threshold as \% predicted maximum oxygen consumption during exercise (VO2; $p<0.05)$, length of high dependency care stay $(p<0.001)$, length of ward stay $(p<0.01)$ and length of hospital stay $(p<0.001)$. There were significant differences between those patients with and without operative anastomotic leak in body mass index $(\mathrm{BMl} ; \mathrm{p}<0.05)$, length of high dependency care stay $(p<0.001)$, length of ward stay (6 vs.15, respectively; $p<0.001)$, length of hospital stay (14 vs. 28 , respectively; $p<0.001)$ and duration between date of surgery and date of commencing postoperative chemotherapy $(p \leq 0.01)$.

Conclusion: CPET and in particular anaerobic threshold \% maximum predicted is useful in predicting the likelihood of the development of pulmonary complications in patients undergoing upper gastrointestinal surgery.
\end{abstract}

Keywords: CPET, surgery, anaerobic threshold, respiratory, risk, lung function, outcome.

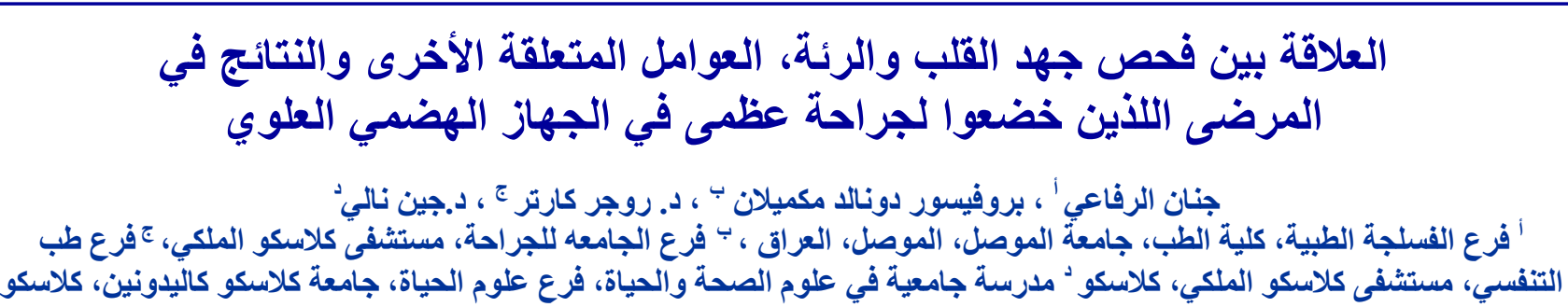




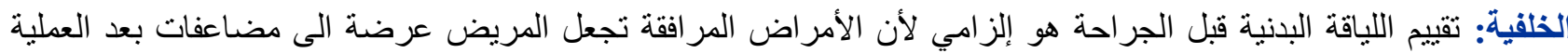

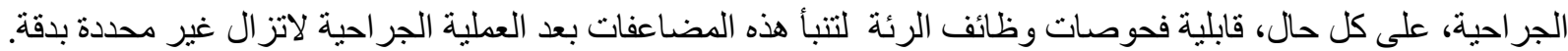

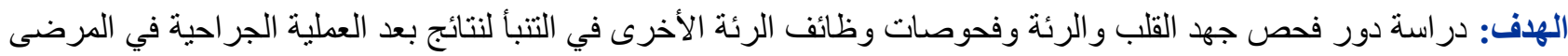
الذين يخضعون لجر احة عظمى في الجهاز الهضمي العلوي التي تعني المعدة و المرئ و الأمعاء الصغيرة و المر التهارة و البنكرياس.

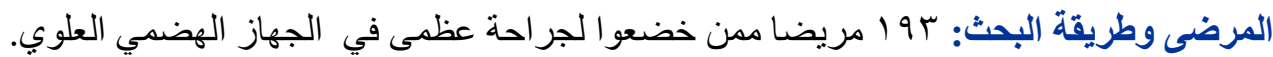

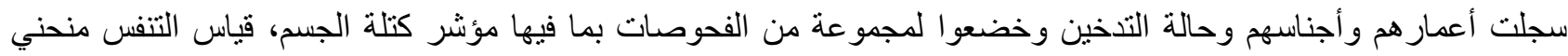

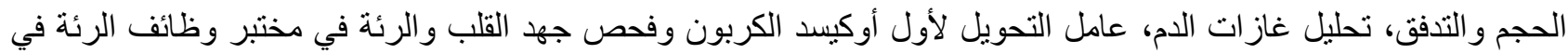

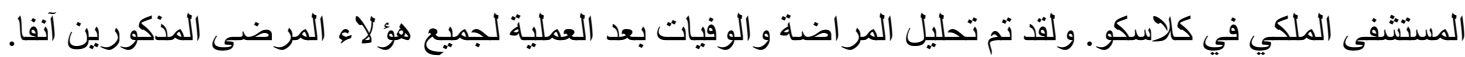

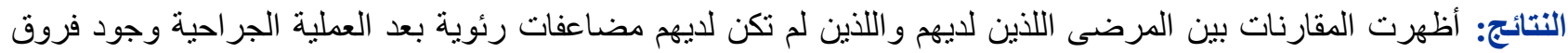

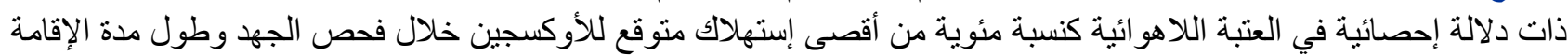

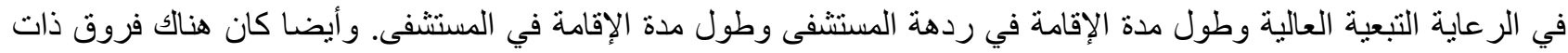

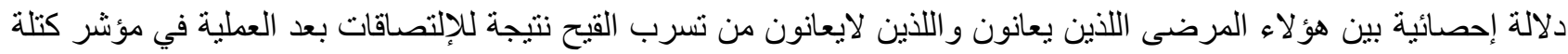

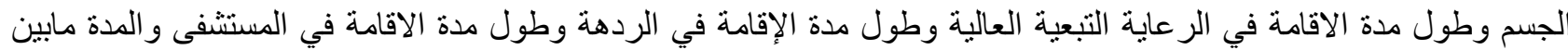

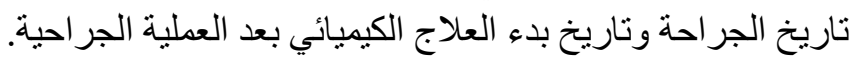

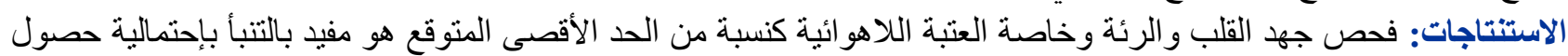

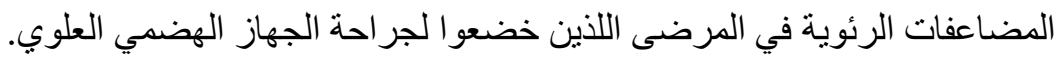

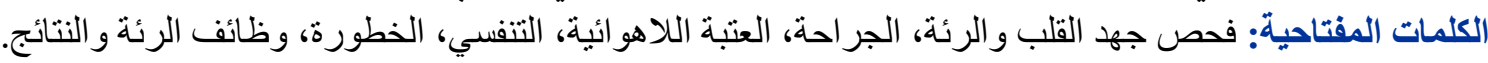

\section{INTRODUCTION}

$\mathrm{D}$ ecreasing complications and death following major surgery remains a significant challenge. Postoperative complications have an enormous influence on early recovery and hospital outcome as well as on long term survival after surgery $^{1,2}$ therefore, identification of vulnerable groups before surgery is important in order to stratify postoperative risk, future intervention studies and ensure the efficient use of resources related to health care ${ }^{2}$.

Outcome following surgery is dependent on two main factors: patient fitness and the operation itself. Postoperative complications most probably occur in a small group of high risk patients; furthermore many patients undergoing gastrooesophageal cancer surgery have major co-morbid disease, which strongly influences their postoperative death risk ${ }^{2,3}$.

Oesophageal and gastric cancer are common diseases that cause great challenges to surgeons because most patients present with advanced disease and curative surgery needs considerable resources in the operating theatre and in critical care $^{3}$.

There is a simple scoring system called the physiological and operative severity score for the enumeration of mortality and morbidity (POSSUM) and this system has proved an efficient indicator of the risk of morbidity and mortality in the general surgical patients. It requires, however, information both pre-operatively and post-operatively ${ }^{4}$ and over-estimates post-operative mortality and morbidity for pancreaticoduodenectomy ${ }^{5,6}$. Other risk stratification tests such as resting spirometry, electrocardiogram and echocardiogram are designed to predict specific organ postoperative complications, mainly respiratory or cardiac ${ }^{7-9}$. Blood gas analysis and anthropometry has been recommended in certain conditions, for example pre-operative respiratory symptoms, major operation, prolonged duration of surgery or when obesity is a risk factor for operation ${ }^{10,11}$. These investigations have been found to be limited predictive tools for more generalised postoperative complications $^{12,13}$. In addition, some of these investigations are often time and resource limited $^{14}$.

Recently, it has been reported that CPET offers an objective measurement of cardiopulmonary fitness that can determine the physiological capacity of the individual to meet the increases in metabolic demands that occur during trauma like 
major surgery ${ }^{15,16}$. There is, however, no clear agreement on the best CPET parameter to predict post-operative outcome. Some studies demonstrated that exercise test variables (Peak $\mathrm{VO}_{2}\left(\mathrm{PVO}_{2}\right)$, anaerobic threshold) can predict post oesophagectomy cardiopulmonary complications in oesophageal cancer patients ${ }^{17,18}$; while another study suggested that CPET was of limited value in predicting cardiopulmonary complication post oesophagectomy ${ }^{19}$. Anaerobic threshold measurement improved the prediction of postnon-cardiopulmonary surgery complications compared with questionnaire-based activity assessment of metabolic equivalent score functional capacity ${ }^{13}$.

\section{Aim of the study}

There have been few comparative studies of the role of CPET in the analysis of peri-operative risk assessment in patients undergoing major upper gastrointestinal surgery and neoadjuvant chemotherapy. The aim of the present study was to examine the relationship between these preoperative measurements and post-operative outcomes in those patients.

\section{PATIENTS AND METHODS}

243 patients were referred to the Pulmonary Function Laboratory of Glasgow Royal Infirmary between August 2008 and December 2011 who subsequently underwent surgery. A total of 193 of these patients underwent major upper gastrointestinal surgery within 60 days post CPET. Age, sex and smoking status were recorded.

A further 19 patients underwent major upper gastrointestinal surgery more than 60 days post CPET, 17 patients underwent abdominal aortic aneurysm surgery, 13 underwent less radical upper gastrointestinal surgery (laparoscopic excision or laparotomy only) and 1 patient's case record was not accessible to evaluate the degree of upper gastrointestinal surgery. These cases were excluded from this study.

Investigations at the pulmonary function laboratory: The diagnosed patients were referred to the pulmonary laboratory. Tests including anthropometric measurements, flow-volume curve spirometry test, blood gas analysis, transfer factor for carbon monoxide test (TLCO) and cardiopulmonary exercise tests (CPET) were performed. CPET was conducted by using an electrically braked bicycle ergometer. An electronic sphygmomanometer and 12- ECG electrodes were connected to the patient and the CPET protocol was started with 3 minutes rest. At the end of 3 minutes, the patients were asked to start unloaded cycling for 1 minute at a constant pedaling rate of between 50-60 revolution per minute (rpm) depending on the metronomic of the bike ${ }^{20,21}$. After that the work load started to increase incrementally of 10-20 watt/minute but it was modified depending on subject's fitness, so when the patient was very frail and old, the increment rate was decreased to $5 \mathrm{watt} / \mathrm{min}$ and when the patient was young and fit, the increment rate was increased to $15 \mathrm{watt} / \mathrm{min}^{22-24}$. The patients continued pedaling until either; they reached their exhaustion level or they had any other limiting symptom that prevent them to continue more cycling ${ }^{25,26}$. At the end point of the test, the patient entered the recovery period where they were asked to continue pedaling without load for at least 2 minutes. ECG, heart rate, blood pressure, respiratory exchange ratio (RER) and pulse oximetry were monitored ${ }^{20}$.

The test was ended before the patients reached exhaustion level or when the patients signaled that they had chest pain or difficulty in breathing. The test was also stopped if there was depression or elevation of the ST segment of the ECG $\geq 2 \mathrm{~mm}$, $T$ wave inversion or sustained ventricular tachycardia $15,27,28$

Anaerobic threshold determination was done using the V-slope method 16,28,29 which was confirmed by ventilatory equivalent, end tidal partial pressure methods [Reviewed 16,28 and respiratory exchange ratio (RER) changes ${ }^{30}$.

Follow up of patients for postoperative outcomes: The prospective follow up of all patients who underwent major surgery was performed by examining the following categories:

Morbidity - defined as complications at 30 days postoperatively including medical (cardiac and pulmonary) complications and /or surgical technique (wound infection and an anastomotic leak) complications.

Length of hospital stay (LOHS), high dependency unit (HDU) stay, intensive care unit (ICU) stay and ward stay during the in-hospital 
period and any readmission to them which happened within 30 days postoperatively. Length of high dependency unit stay and length of intensive care stay were merged in this study as length of high dependency care stay.

Mortality - defined as 30 day postoperatively or postoperative in-hospital mortality ${ }^{3}$. The duration between the date of operation and the date of commencing post-operative chemotherapy when it was indicated.

Statistics: The data were presented as median and range or numbers and percentages. An appropriate comparison of patient groups was carried out using a Kruskal-Wallis test, MannWhitney and chi square test from SPSS software; significant was defined as $\mathrm{P}$ value $<0.05$ (twosided).

\section{RESULTS}

The 193 patients were categorised depending on development of post operative cardiopulmonary complications into patients with no post-operative cardiopulmonary complications and those with post operative cardiopulmonary complications. $54 \%$ of patients developed cardiopulmonary complications. Table 1.

Table 1: The relationship between clinicopathological characteristic indices and CPET parameters in patients having upper gastrointestinal surgery in the $1^{\text {st }} 60$ days post CPET grouped into those with and without post-operative cardiopulmonary complications $(n=193)$.

\begin{tabular}{|c|c|c|c|c|c|}
\hline & \multirow{2}{*}{ Parameters } & \multirow{2}{*}{$\begin{array}{l}\text { Normal } \\
\text { range }\end{array}$} & \multicolumn{2}{|c|}{ Cardiopulmonary complication } & \multirow{2}{*}{$\begin{array}{c}\text { P- } \\
\text { value }\end{array}$} \\
\hline & & & No $(n=89)$ & Yes $(n=104)$ & \\
\hline \multicolumn{3}{|l|}{ Age (years) } & $67(38-83)$ & $66(38-86)$ & 0.705 \\
\hline Sex: n (\%) & Male/female & & $50(56) / 39(44)$ & $71(68) / 33(32)$ & 0.084 \\
\hline Smoking & Non-smoker & & $32(42)$ & $28(31)$ & \\
\hline status & Ex smoker & & $25(33)$ & $28(31)$ & \\
\hline $\mathrm{n}(\%)$ & Current smoker & & $19(25)$ & $34(38)$ & 0.065 \\
\hline & Most deprivation (1) & & $20(24)$ & $31(34)$ & \\
\hline Deprivation & & & $13(16)$ & $19(21)$ & \\
\hline Level & & & $21(26)$ & 17 (19) & 0.081 \\
\hline $\mathrm{n}(\%)$ & & & $11(13)$ & $11(12)$ & \\
\hline & Least deprivation (5) & & $17(21)$ & $13(14)$ & \\
\hline \multicolumn{2}{|c|}{ Body mass index $\left(\mathrm{kg} / \mathrm{m}^{2}\right)$} & $18.5-25$ & $25.6(17.6-42.2)$ & $25.4(17.1-39.3)$ & 0.500 \\
\hline \multicolumn{2}{|c|}{ arterial PCO2 (kpa) } & $4.5-6.0$ & $4.4(3.2-5.7)$ & $4.6(3.5-6.9)$ & 0.104 \\
\hline \multicolumn{2}{|l|}{$\mathrm{t} \mathrm{Hb}(\mathrm{g} / \mathrm{dl})$} & $12-16$ & $14(9-17)$ & $14(8-18)$ & 0.488 \\
\hline \multicolumn{2}{|c|}{ carbox_Hb (\%) } & $0.3-1.0$ & $2.6(0.9-10.5)$ & $2.7(1.3-12.5)$ & 0.147 \\
\hline \multicolumn{2}{|c|}{ FVC (\%predicted) } & $>75$ & $118(68-156)$ & $110(62-160)$ & 0.008 \\
\hline \multicolumn{2}{|c|}{ FEV1/FVC (\%Predicted) } & $>75$ & $94(62-122)$ & $96(56-120)$ & 0.563 \\
\hline \multicolumn{2}{|c|}{$\begin{array}{l}\text { TLCO corrected for Hb \& SA (\%predicted) } \\
(n=71)\end{array}$} & $>75$ & $89(59-122)$ & $88(30-120)$ & 0.579 \\
\hline \multicolumn{2}{|c|}{ Peak VO 2 (absolute) $(\mathrm{ml} / \mathrm{min} / \mathrm{kg})$} & $35-40$ & $16.0(10.2-32.0)$ & $15.3(9.2-39.4)$ & 0.876 \\
\hline \multicolumn{2}{|c|}{$\mathrm{PeakVO}_{2}$ (\% predicted maximumVO2) } & $>80$ & $72.1(33.4-128.7)$ & $67.2(38.8-142.7)$ & 0.077 \\
\hline \multicolumn{2}{|c|}{ Anaerobic threshold (absolute)(ml/min/kg) } & $15-25$ & $10.7(6.0-16.9)$ & $10.1(6.3-18.8)$ & 0.502 \\
\hline \multicolumn{2}{|c|}{$\begin{array}{l}\text { Anaerobic threshold (\% predicted } \\
\text { maximum } \mathrm{VO}_{2} \text { ) }\end{array}$} & $40-80$ & $47.2(19.4-83.6)$ & $41.4(24.7-84.5)$ & 0.020 \\
\hline \multicolumn{2}{|c|}{ VE/ $\mathrm{VCO}_{2}$ (at anaerobic threshold) } & $23-34$ & $30(22-41)$ & $29(21-49)$ & 0.722 \\
\hline \multicolumn{2}{|c|}{ VE/VO 2 (at anaerobic threshold) } & $\leq 34$ & $29(18-43)$ & $28(17-48)$ & 0.759 \\
\hline \multicolumn{2}{|c|}{ Work efficiency (ml/kg.min) } & $8.5-11$ & $9.4(6.1-14.7)$ & $9.5(5.7-13.9)$ & 0.730 \\
\hline \multicolumn{2}{|c|}{ Ventilatory reserve (\%) } & $15-50$ & $44(20-83)$ & $46(16-91)$ & 0.768 \\
\hline \multicolumn{2}{|c|}{ Length of high dependency care stay } & & $7(2-15)$ & $9(3-59)$ & $<0.001$ \\
\hline \multicolumn{2}{|c|}{ Length of ward stay } & & $5(0-28)$ & $9(0-48)$ & $<0.001$ \\
\hline \multicolumn{2}{|c|}{ Length of hospital stay } & & $12(6-39)$ & $19(7-80)$ & $<0.001$ \\
\hline \multicolumn{2}{|c|}{$\begin{array}{l}1^{\text {st }} \text { postoperative month mortality: } \\
\text { No }[\mathrm{n}(\%)] / \text { yes }[\mathrm{n}(\%)]\end{array}$} & & $89(100) / 0(0)$ & $94(90) / 10(10)$ & 0.003 \\
\hline \multicolumn{2}{|c|}{$\begin{array}{l}\text { Duration between surgery and commencing } \\
\text { postoperative chemotherapy }\end{array}$} & & $69(37-268)$ & $73(37-887)$ & 0.219 \\
\hline
\end{tabular}


There were significant associations between postoperative cardiopulmonary complications and mortality within the first month postoperatively or in-hospital mortality $(p \leq 0.01)$, where patients with post-operative cardiopulmonary complications had a higher percentage of mortality compared with patients with no post-operative cardiopulmonary complications. There were significant difference between the groups with and without post operative cardiopulmonary complications in FVC \% predicted (118 vs. 110 , respectively; $p \leq 0.01$ ), anaerobic threshold \% predicted maximum $\mathrm{VO}_{2}$ (47.2 vs. 41.4 , respectively; $p<0.05$ ), length of high dependency care stay ( 7 vs. 9 , respectively; $p<0.001)$, in length of ward stay (5 vs. 9 , respectively; $p<0.001)$ and length of hospital stay (12 vs. 19 , respectively; $p<0.001$ ). There were no significant differences between the two groups in other variables.

The 193 patients who underwent major upper gastrointestinal surgery within 60 days post CPET were then categorised depending on development of post operative pulmonary complications into two groups; those with no post operative pulmonary complications those with post operative pulmonary complications. $44 \%$ of patients developed postoperative pulmonary complications. Table 2.

Table 2: The relationship between clinicopathological characteristic indices and CPET parameters in patients having upper gastrointestinal surgery in the $1^{\text {st }} 60$ days post CPET grouped into those with and without post-operative pulmonary complications $(\mathrm{n}=193)$.

\begin{tabular}{|c|c|c|c|c|c|}
\hline & \multirow{2}{*}{ Parameters } & \multirow{2}{*}{$\begin{array}{l}\text { Normal } \\
\text { range }\end{array}$} & \multicolumn{2}{|c|}{ Pulmonary complication } & \multirow{2}{*}{ P-value } \\
\hline & & & No $(n=109)$ & Yes $(n=84)$ & \\
\hline \multicolumn{2}{|l|}{ Age (years) } & & $67(38-85)$ & $64(39-86)$ & 0.320 \\
\hline Sex: $n(\%)$ & Male/female & & $67(61) / 42(39)$ & $54(64) / 30(36)$ & 0.689 \\
\hline Smoking & Non-smoker & & $40(43)$ & $20(28)$ & \\
\hline status & Ex smoker & & $31(33)$ & $22(30)$ & \\
\hline $\mathrm{n}(\%)$ & Current smoker & & $23(24)$ & $30(42)$ & 0.014 \\
\hline & Most deprivation (1) & & $23(23)$ & $28(38)$ & \\
\hline Deprivation & (2) & & $19(19)$ & $13(18)$ & \\
\hline Level & & & $24(24)$ & $14(19)$ & 0.095 \\
\hline $\mathrm{n}(\%)$ & (4) & & $15(16)$ & $7(9)$ & \\
\hline & Least deprivation (5) & & $18(18)$ & $12(16)$ & \\
\hline \multicolumn{2}{|c|}{ Body mass index $\left(\mathrm{kg} / \mathrm{m}^{2}\right)$} & $18.5-25$ & $25.9(17.6-42.2)$ & $25.2(17.1-37.6)$ & 0.842 \\
\hline \multicolumn{2}{|c|}{ arterial $\mathrm{PCO}_{2}(\mathrm{kpa})$} & $4.5-6.0$ & $4.5(3.2-6.9)$ & $4.6(3.6-6.3)$ & 0.142 \\
\hline \multicolumn{2}{|l|}{$\mathrm{t} \mathrm{Hb}(\mathrm{g} / \mathrm{dl})$} & $12-16$ & $14(9-17)$ & $14(8-18)$ & 0.531 \\
\hline \multicolumn{2}{|c|}{ carbox_Hb (\%) } & $0.3-1.0$ & $2.5(0.9-10.5)$ & $2.8(1.3-12.5)$ & 0.088 \\
\hline \multicolumn{2}{|c|}{ FVC (\% predicted) } & $>75$ & $118(68-156)$ & $109(62-160)$ & 0.002 \\
\hline \multicolumn{2}{|c|}{ FEV1/FVC (\% Predicted) } & $>75$ & $95(62-122)$ & $96(56-120)$ & 0.423 \\
\hline \multicolumn{2}{|c|}{ TLCO corrected for Hb \& SA (\% predicted) $(n=71)$} & $>75$ & $89(59-122)$ & $88(30-120)$ & 0.435 \\
\hline \multicolumn{2}{|c|}{ Peak $\mathrm{VO}_{2}$ (absolute)(ml/min/kg) } & $35-40$ & $16.2(10.2-34.3)$ & $15.1(9.2-39.4)$ & 0.428 \\
\hline \multicolumn{2}{|c|}{ PeakVO2 (\% predicted maximumVO2) } & $>80$ & $72.0(33.4-128.7)$ & $67.2(39.4-142.7)$ & 0.083 \\
\hline \multicolumn{2}{|c|}{ Anaerobic threshold (absolute)(ml/min/kg) } & $15-25$ & $10.7(6.0-16.9)$ & $9.8(6.3-18.8)$ & 0.114 \\
\hline \multicolumn{2}{|c|}{$\begin{array}{l}\text { Anaerobic threshold } \\
\text { (\% predicted maximumVO2 ) }\end{array}$} & $40-80$ & $47.2(19.4-83.6)$ & $40.4(25.1-84.5)$ & 0.019 \\
\hline \multicolumn{2}{|c|}{$\mathrm{VE} / \mathrm{VCO}_{2}$ (at anaerobic threshold) } & 23-34 & $30(21-41)$ & $29(21-49)$ & 0.514 \\
\hline \multicolumn{2}{|c|}{ VE/VO2 (at anaerobic threshold) } & $\leq 34$ & $29(18-43)$ & $28(17-48)$ & 0.369 \\
\hline \multicolumn{2}{|c|}{ Work efficiency (ml/kg.min) } & $8.5-11$ & $9.3(5.9-14.7)$ & $9.6(5.7-13.9)$ & 0.314 \\
\hline \multicolumn{2}{|c|}{ Ventilatory reserve (\%) } & $15-50$ & $45(20-83)$ & $45(16-91)$ & 0.725 \\
\hline \multicolumn{2}{|c|}{ Length of high dependency care stay } & & $7(2-16)$ & $10(4-59)$ & $<0.001$ \\
\hline \multicolumn{2}{|c|}{ Length of ward stay } & & $5(0-28)$ & $9(0-48)$ & 0.002 \\
\hline \multicolumn{2}{|c|}{ Length of hospital stay } & & $12(6-40)$ & $19(7-80)$ & $<0.001$ \\
\hline \multicolumn{2}{|c|}{$\begin{array}{l}1^{\text {st }} \text { postoperative month mortality: } \\
\text { No }[\mathrm{n}(\%)] / \text { yes [n (\%)] }\end{array}$} & & $109(100) / 0(0)$ & $74(88) / 10(12)$ & $<0.001$ \\
\hline \multicolumn{2}{|c|}{$\begin{array}{l}\text { Duration between surgery and commencing } \\
\text { postoperative chemotherapy }\end{array}$} & & $70(37-268)$ & $72(37-887)$ & 0.620 \\
\hline
\end{tabular}

*All values given as median (range) or numbers (\%). Total patients' numbers vary due to incomplete data.

$\mathrm{SA}=$ surface area; $\mathrm{VE}=$ ventilatory equivalent. 
There were significant associations between postoperative pulmonary complications with smoking status $(p \leq 0.05)$, where patients without postoperative pulmonary complications had a higher percentage of non-smokers and lower percentage of current smokers compared with those with postoperative pulmonary complications. There were significant associations between postoperative pulmonary complications and mortality within the first month postoperatively or in-hospital mortality $(p<0.001)$, where patients with post-operative pulmonary complications had higher mortality levels compared with patients without post-operative pulmonary complications. There were significant differences between without and those with post operative pulmonary complications in FVC \% predicted (118 vs. 109, respectively; $\mathrm{p} \leq 0.01$ ), in anaerobic threshold \% predicted maximum $\mathrm{VO}_{2}$ (47.2 vs. 40.4 , respectively; $p<0.05$ ), in length of high dependency care stay ( 7 vs. 10 , respectively; $p<0.001)$, in length of ward stay (5 vs. 9 , respectively; $p \leq 0.01$ ) and in length of hospital stay (12 vs. 19 , respectively; $p<0.001$ ). There were no significant differences between the two groups in other variables.

The 193 patients were then further categorised depending on the development of post operative anastomotic leak complications into two groups: Those without post operative anastomotic leak and those patients with post operative anastomotic leak. $16 \%$ of patients developed post-operative anastomotic leak. Table 3.

Table 3: The relationship between clinicopathological characteristic indices, CPET parameters in 1st 60 days in patients with and without postoperative anastomotic leak in upper gastrointestinal surgery $(n=193)$.

\begin{tabular}{|c|c|c|c|c|c|}
\hline & \multirow{2}{*}{ Parameters } & \multirow{2}{*}{$\begin{array}{l}\text { Normal } \\
\text { range }\end{array}$} & \multicolumn{2}{|c|}{ An anastomotic leak } & \multirow{2}{*}{$\begin{array}{c}\text { P- } \\
\text { value }\end{array}$} \\
\hline & & & No $(n=162)$ & Yes $(n=31)$ & \\
\hline Age (years) & & & $66(38-86)$ & $67(39-83)$ & 0.718 \\
\hline Sex: $n(\%)$ & Male/female & & $100(62) / 62(38)$ & $21(68) / 10(32$ & 0.527 \\
\hline Smoking & Non-smoker & & $51(37)$ & $9(32)$ & \\
\hline status & Ex smoker & & $44(32)$ & $9(32)$ & 0.584 \\
\hline $\mathrm{n}(\%)$ & Current smoker & & $43(31)$ & $10(36)$ & 0.004 \\
\hline Deprivation & Most deprivation (1) & & $43(30)$ & $8(29)$ & \\
\hline Level & (2) & & $25(17)$ & $7(25)$ & \\
\hline$n(\%)$ & (3) & & $35(24)$ & $3(11)$ & 0.934 \\
\hline & & & $16(11)$ & $6(21)$ & \\
\hline & Least deprivation (5) & & $26(18)$ & $4(14)$ & \\
\hline Body mass it & $x\left(\mathrm{~kg} / \mathrm{m}^{2}\right)$ & $18.5-25$ & $25.2(17.1-42.2)$ & $27.2(18.7-37.6)$ & 0.044 \\
\hline arterial $\mathrm{PCO}$ & pa) & $4.5-6.0$ & $4.5(3.2-6.9)$ & $4.7(3.4-6.3)$ & 0.034 \\
\hline t Hb (g/dl) & & $12-16$ & $14(8-18)$ & $14(10-18)$ & 0.521 \\
\hline carbox_Hb ( & & $0.3-1.0$ & $2.6(0.9-12.5)$ & $2.6(1.5-6.9)$ & 0.782 \\
\hline FVC $(\%$ prec & ed) & $>75$ & $114(62-160)$ & $108(70-150)$ & 0.102 \\
\hline FEV1/FVC (c & Predicted) & $>75$ & $94(58-122)$ & $97(56-117)$ & 0.202 \\
\hline TLCO correcte & or $\mathrm{Hb} \& \mathrm{SA}$ (\%predicted) $(\mathrm{n}=71)$ & $>75$ & $89(30-122)$ & $88(42-120)$ & 0.612 \\
\hline Peak $\mathrm{VO}_{2}(\mathrm{a}$ & lute)(ml/min/kg) & $35-40$ & $16.0(9.2-39.4)$ & $15.0(10.2-26.8)$ & 0.204 \\
\hline $\mathrm{PeakVO}_{2}(\%$ & edicted maximumVO2) & $>80$ & $70.2(33.4-142.7)$ & $67.9(34.5-131.9)$ & 0.200 \\
\hline Anaerobic th & hold (absolute)(ml/min/kg) & $15-25$ & $10.6(6.0-18.8)$ & $9.8(6.7-16.6)$ & 0.274 \\
\hline $\begin{array}{l}\text { Anaerobic thre } \\
\text { (\% predicted } n\end{array}$ & $\begin{array}{l}\text { old } \\
\text { imumVO2 ) }\end{array}$ & $40-80$ & $45.6(19.7-83.6)$ & $42.1(19.4-84.5)$ & 0.241 \\
\hline VE $/ \mathrm{VCO}_{2}$ (at & aerobic threshold) & $23-34$ & $30(22-49)$ & $28(21-37)$ & 0.084 \\
\hline $\mathrm{VE} / \mathrm{VO}_{2}($ at a & erobic threshold) & $\leq 34$ & $29(18-48)$ & $27(17-37)$ & 0.096 \\
\hline Work efficien & (ml/kg.min) & $8.5-11$ & $9.4(5.7-14.7)$ & $9.6(6.3-11.7)$ & 0.581 \\
\hline Ventilatory re & rve (\%) & $15-50$ & $46(16-91)$ & $45(24-74)$ & 0.694 \\
\hline Length of hig & dependency care stay & & $7(2-42)$ & $14(5-59)$ & $<0.001$ \\
\hline Length of wa & stay & & $6(0-40)$ & $15(0-48)$ & $<0.001$ \\
\hline Length of ho & tal stay & & $14(6-70)$ & $28(13-80)$ & $<0.001$ \\
\hline $1^{\text {st }}$ postopera & e month mortality & & $159(98) / 3(2)$ & $24(77) / 7(23)$ & $<0.001$ \\
\hline $\begin{array}{l}\text { Duration betw } \\
\text { postoperative }\end{array}$ & $\begin{array}{l}\text { surgery and commencing } \\
\text { motherapy }\end{array}$ & & $68(37-887)$ & $101(64-143)$ & 0.007 \\
\hline
\end{tabular}


There were significant associations between post-operative anastomotic leak and mortality within the first month postoperatively or in hospital mortality $(p<0.001)$, where patients with postoperative anastomotic leak had a higher percentage of mortality compared with patients without. There were significant differences between patients without and those with post operative anastomotic leak in body mass index (25.2 vs. 27.2, respectively; $\mathrm{p}<0.05$ ), in $\mathrm{PCO} 2$ (kpa) (4.5 vs. 4.7 , respectively; $p<0.05$ ), in length of high dependency care stay ( 7 vs. 14 , respectively; $p<0.001$ ), in length of ward stay ( 6 vs.15, respectively; $p<0.001$ ), in length of hospital stay (14 vs. 28 , respectively; $p<0.001$ ) and in duration between date of surgery and date of commencing postoperative chemotherapy (68 vs. 101, respectively; $p \leq 0.01)$. There were no significant differences between both groups in other variables.

\section{DISCUSSION}

The results of the present study show that CPET was an indicator of the development of postoperative complications. In particular, a reduction in anaerobic threshold \% predicted maximum $\mathrm{VO}_{2}$ was associated with post-operative cardiopulmonary complications and in particular pulmonary complications.

This finding is supported by other studies demonstrating that pre-operative CPET and especially anaerobic threshold measurement can predict patients at risk for early postoperative complications ${ }^{31,13}$. Conversely, other studies have suggested that CPET has a limited value in predicting post-operative cardiopulmonary morbidity in patients undergoing oesophagectomy 19. Forshaw et al. used a small patient number (64), male patients only and had a smaller proportion of patients (16\%) with an anaerobic threshold less than $11 \mathrm{ml} / \mathrm{min} / \mathrm{kg}$ that underwent an operation. The present study had a larger sample size (193), examined both sexes and had $64 \%$ of patients with an anaerobic threshold less than 11 $\mathrm{ml} / \mathrm{min} / \mathrm{kg}$ undergoing an operation which may explain the differences. Our results are also in contrast with those of Paisani et al. (2012) who suggested that cardiopulmonary assessment using the 6-minute walking test pre-operatively did not predict post-operative pulmonary complications in upper abdominal surgery ${ }^{32}$. Other authors have described the 6-minute walking test as a useful tool to stratify risk where CPET is unavailable ${ }^{33}$, however the difference between the results of Paisani, et al. and the present study suggest that CPET is a more powerful tool in surgical risk assessment.

Major surgery places a severe stress on the cardiopulmonary reserve. This is associated with an increase in oxygen consumption by $40 \%$, and this increase has to be continued for several days post surgery, so that a substantially greater cardiorespiratory reserve is required ${ }^{34,35}$. The rationale for investigating cardiopulmonary exercise test is based on the fact that CPET is as an indicator of cardiopulmonary reserve. This study found that an anaerobic threshold determined by CPET would be a more precise variable to predict cardiopulmonary reserve than $\mathrm{PVO}_{2}$ because anaerobic threshold is not dependent on patient motivation ${ }^{36}$. In addition, oxygen extraction rate during exercise is much greater than that extracted during surgery ${ }^{35}$. This finding of ability of anaerobic threshold to predict cardiopulmonary reserve better than $\mathrm{PVO}_{2}$ is, however, not supported by Nagamatsu et al., who suggested that $\mathrm{VO}_{2}$ maximum closely correlated with the occurrence of post-operative cardiopulmonary complications in oesophagectomy, while the anaerobic threshold did not correlate with the occurrence of complications ${ }^{18}$. The difference in results may be due to the Japanese population examined by Nagamatsu et al. being different from the Glasgow population used in the present study.

FVC\% predicted may play an important role in predicting post-operative cardiopulmonary complications and in particular pulmonary complications. This finding is consistent with the recent study that outlined that FVC can predict $5 \mathrm{yr}$ survival after major upper gastrointestinal surgery 37. Interestingly, this finding in this study was observed in patients with normal pulmonary function, in particular FVC \% predicted. The physiological explanation of this finding is that all patients who undergo abdominal surgery have some impairment of respiratory function and in addition, anaesthesia causes the development of areas of microatelectasis, which grow due to shallow monotonous ventilation, along with 
reduced mucociliary clearance that accompanies postoperative lethargy. Furthermore anaesthesia leads to a temporary dysfunction of the diaphragm after abdominal surgery which helps to explain the affinity of atelectasis for the bases of the lungs ${ }^{38}$. This respiratory muscle dysfunction and microatelectasis may increase the frequency of postoperative pulmonary complications. In addition, the presence of either preoperative obstructive or restrictive pulmonary disease increases the risk of developing perioperative respiratory complications ${ }^{39,40}$, so that postoperative pulmonary complication can occur in patients with normal preoperative lung function and not necessarily only in patients with reduced lung function.

In this study post-operative pulmonary complications were the most common complications (44\%). This finding is supported by previous work that has suggested that pulmonary complications may be as or more common than cardiac complications [Reviewed by 41]. It has been found that there is a wide estimation of postoperative pulmonary complications in the literature $(20-69 \%)^{13,42,43}$. This study found that the pulmonary complication frequency was approximately $44 \%$ which is located within this range.

In the present study it was showed that CPET was not helpful to predict an anastomotic leak. This finding was inconsistent with the study that demonstrated that CPET and especially anaerobic threshold were associated with post-operative pancreatic leakage ${ }^{44}$. In the Ausania et al., study, however, patients underwent only one kind of surgery (pancreaticoduodenectomy) while in this study, the patients underwent different upper gastrointestinal surgeries.

There was a positive relationship between BMI and postoperative anastomotic leak in the present study. This is in keeping with a previous study which showed that patients with higher BMI have a more likelihood to develop gastrointestinal leak post laparoscopic ROUX-en-Y gastric bypass and post gastrectomy ${ }^{45,46}$.

All types of complications, including cardiopulmonary, pulmonary and anastomotic leak complications led to secondary outcomes including increasing in length of high dependency care stay, ward stay and length of hospital stay as well as increased first postoperative month mortality, this finding is supported by many studies 21 , Reviewed by $41,47,48,49$. Anastomotic leak had an additional secondary outcome which was an increase in the duration between operation and the date of commencing chemotherapy where chemotherapy was indicated. This is consistent with other studies which have shown that the likelihood of receiving adjuvant treatment is negatively affected by the course of postoperative recovery in pancreatic cancer surgery ${ }^{50,51}$.

\section{CONCLUSION}

It appear from the results of the present study that CPET, especially AT \% maximum predicted, is the useful one of CPET parameters in predicting the likelihood of development cardiopulmonary complication and in particular pulmonary complications in patients undergoing upper gastrointestinal surgery.

\section{REFERENCES}

1. Khuri SF, Henderson WG, DePalma RG, Mosca C, Healey NA, Kumbhani DJ. Determinants of long-term survival after major surgery and the adverse effect of postoperative complications. Annals of Surgery 2005; $242(3): 326$.

2. Pearse RM, Harrison DA, James $P$, Watson D, Hinds $\mathrm{C}$, Rhodes $\mathrm{A}$, et al. Identification and characterisation of the high-risk surgical population in the united kingdom. Crit Care 2006; 10 (3): R81.

3. McCulloch P, Ward J, Tekkis PP. Mortality and morbidity in gastro-oesophageal cancer surgery: Initial results of ASCOT multicentre prospective cohort study. British Medical Journal 2003; 327 (7425):1192.

4. Copeland GP. The POSSUM system of surgical audit. Archives of Surgery 2002; 137 (1):15.

5. Copeland G, Jones D, Walters M. POSSUM: A scoring system for surgical audit. British Journal of Surgery 1991; 78(3): 355-360.

6. Khan AW, Shah SR, Agarwal AK, Davidson BR Evaluation of the POSSUM scoring system for comparative audit in pancreatic surgery. Digestive Surgery 2003; 20 (6): 539-545.

7. Halm EA, Browner WS, Tubau JF, Tateo IM, Mangano DT. Echocardiography for assessing cardiac risk in patients having noncardiac surgery. Annals of Internal Medicine 1996;125 (6) :433.

8. Lee TH, Marcantonio ER, Mangione CM, Thomas EJ, Polanczyk CA, Cook EF, et al. Derivation and prospective validation of a simple index for prediction of cardiac risk of major noncardiac surgery. Circulation 1999;100 (10): 1043-1049.

9. McAlister FA, Bertsch K, Man J, Bradley J , Jacka M. Incidence of and risk factors for pulmonary complications after nonthoracic surgery. American Journal of Respiratory and Critical Care Medicine 2005; 171(5):514-517. 
10. Khan MA, Hussain SF. Pre-operative pulmonary evaluation. J Ayub Med Coll Abbottabad 2005;17(4):8286.

11. Khoury W, Stocchi L, Geisler D. Outcomes after laparoscopic intestinal resection in obese versus nonobese patients. British Journal of Surgery 2011; 98(2): 293-298.

12. Smetana GW. Preoperative pulmonary assessment of the older adult. Clinics in Geriatric Medicine 2003; 19(1): 35-56.

13. Snowden CP, Prentis JM, Anderson HL, et al. Submaximal cardiopulmonary exercise testing predicts complications and hospital length of stay in patients undergoing major elective surgery. ann surg 2010; 251(3):535-541.

14. Chassot PG, Delabays A, Spahn D. Preoperative evaluation of patients with, or at risk of, coronary artery disease undergoing non-cardiac surgery. British Journal of Anaesthesia 2002; 89 (5):747-759.

15. Struthers $R$, Erasmus $P$, Holmes $K$, Warman $P$, Collingwood A, Sneyd J. Assessing fitness for surgery: A comparison of questionnaire, incremental shuttle walk, and cardiopulmonary exercise testing in general surgical patients. British Journal of Anaesthesia 2008; 101(6): 774

16. Smith $T$, Stonell $C$, Purkayastha $S$, Paraskevas $P$. Cardiopulmonary exercise testing as a risk assessment method in non cardio-pulmonary surgery: A systematic review. Anaesthesia 2009; 64(8):883-893(abstract).

17. Nagamatsu $Y$, Yamana $H$, Fujita $H$, et al. The simultaneous evaluation of preoperative cardiopulmonary functions of oesophageal cancer patients in the analysis of expired gas with exercise testing. Nippon Kyobu Geka Gakkai Zasshi 1994;42: pp. 2037-40(abstract) Article in Japanese.

18. Nagamatsu $Y$, Shima I, Yamana $H$, Fujita $H$, Shirouzu K, Ishitake T. Preoperative evaluation of cardiopulmonary reserve with the use of expired gas analysis during exercise testing in patients with squamous cell carcinoma of the thoracic esophagus. The Journal of Thoracic and Cardiovascular Surgery 2001; 121(6): 1064.

19. Forshaw MJ, Strauss DC, Davies AR, Wilson D, Lams B, Pearce A, et al. Is cardiopulmonary exercise testing a useful test before esophagectomy?. The Annals of Thoracic Surgery 2008; 85 (1) : 294-299.

20. Epstein SK, Freeman RB, Khayat A, Unterborn JN, Pratt DS, Kaplan MM. Aerobic capacity is associated with 100-day outcome after hepatic transplantation. Liver Transplantation2004; 10(3): 418-424.

21. Older $P$, Smith $R$, Courtney $P$, Hone R. Preoperative evaluation of cardiac failure and ischemia in elderly patients by cardiopulmonary exercise testing. Chest 1993; 104 (3): 701-704.

22. Weisman IM ZR. An integrated approach to the interpretation of CPET. in: Clinical exercise testing. Weisman IM, Zeballos RJ, eds. ; edn, Philadelphia, W.B. Saunders, Clinics in ChestMedicine1994.

23. Wasserman K, Hansen JE, Sue DY, Whipp BJ, Casaburi. Principles of exercise testing and interpretation. in: Philadelphia, Lea \& Febiger 1994.

24. Whipp B. Exercise testing:The how and the why of special edition: Cardiopulmonary exercise testing information, diagnosis,essay, VIASYS Info Special Edition CPET, $1^{\text {st }}$ edition, April 2002: 1-11.

25. Casaburi R, Cotes J, Donner C, Estenne M, Folgering $\mathrm{H}$, Higenbottam $\mathrm{T}$, et al. Clinical exercise testing with reference to lung diseases: Indications, standardization and interpretation strategies. Eur Respir J 1997;10: 2662-2689.

26. Gitt AK, Wasserman K, Kilkowski C, Kleemann T, Kilkowski $\mathrm{A}$, Bangert $\mathrm{M}$, et al. Exercise anaerobic threshold and ventilatory efficiency identify heart failure patients for high risk of early death. Circulation 2002; 106(24): 3079.

27. McCullough PA, Gallagher MJ, dejong AT, Sandberg $\mathrm{KR}$, Trivax JE, Alexander D, et al. Cardiorespiratory fitness and short-term complications after bariatric surgery. Chest 2006;130(2):517.

28. American Thoracic Society \& American College of Chest Physicians ATS/ACCP statement on cardiopulmonary exercise testing. Am J Respir Crit Care Med 2003; 167: 211-277.

29. Beaver W.L, Wasserman K, Whipp B.J. Bicarbonate buffering of lactic acid generated during exercise. Journal of Applied Physiology 1986; 60(2): 472-478 (abstract).

30. Weisman IM , Zeballos RJ (eds). An integrative approach to the interpretation of cardiopulmonary exercise testing 2002(online).

31. Older P, Smith R, Hall A, French C. Preoperative cardiopulmonary risk assessment by cardiopulmonary exercise testing. Critical Care and Resuscitation 2000;2: 198-208.

32. Paisani DM, Fiore JF, Lunardi AC, Colluci DBB, Santoro IL, Carvalho CRF, et al. Preoperative Six-Minute walking distance does not predict pulmonary complications in upper abdominal surgery. Respirology (Wiley Online Library ) 2012(abstract).

33. Sinclair R, Batterham A, Davies S, Cawthorn L, Danjoux G. Validity of the 6 min walk test in prediction of the anaerobic threshold before major non-cardiac surgery. British Journal of Anaesthesia 2012; 108(1): 3035.

34. Older $\mathrm{P}$, Smith $\mathrm{R}$. Experience with the preoperative invasive measurement of haemodynamic, respiratory and renal function in 100 elderly patients scheduled for major abdominal surgery. Anaesthesia and Intensive Care 1988; 16(4) : 389-395.

35. Agnew M. Spirometry in clinical use: Practical issues. Breathe2010;6: 196-203.

36. Kothmann E, Danjoux G, Owen S, Parry A, Turley A \& Batterham A. Reliability of the anaerobic threshold in cardiopulmonary exercise testing of patients with abdominal aortic aneurysms*. Anaesthesia 2009; 64(1): 9-13.

37. Colson M, Baglin J, Bolsin S, Grocott M. Cardiopulmonary exercise testing predicts $5 \mathrm{yr}$ survival after major surgery. British Journal of Anaesthesia 2012 109(5):735-741.

38. Hall JC, Tarala RA, Tapper J, Hall JL. Prevention of respiratory complications after abdominal surgery: A randomised clinical trial. Bmj 1996;312(7024): 148-152. 39. Fleisher LA, Beckman JA, Brown KA, Calkins $H$, Chaikof EL, Fleischmann KE, et al. ACC/AHA 2007 guidelines on perioperative cardiovascular evaluation and care for noncardiac surgery: Executive summary: A 
report of the american college of Cardiology/American heart association task force on practice guidelines (writing committee to revise the 2002 guidelines on perioperative cardiovascular evaluation for noncardiac surgery). Circulation 2007; 116(17): 1971.

40. Kanat F, Golcuk A, Teke T, Golcuk M. Risk factors for postoperative pulmonary complications in upper abdominal surgery. ANZ Journal of Surgery 2007; 77(3): 135-141.

41. Lawrence VA, Dhanda R, Hilsenbeck SG, Page CP. Risk of pulmonary complications after elective abdominal surgery. Chest 1996; 110(3) 744.

42. Hall J, Tarala R, Hall J, Mander J. A multivariate analysis of the risk of pulmonary complications after laparotomy. CHEST Journal 1991; 99(4): 923-927.

43. Brooks-Brunn JA. Predictors of postoperative pulmonary complications following abdominal surgery. Chest1997; 111(3): 564-571.

44. Ausania F, Snowden C, Prentis J, Holmes L, Jaques $\mathrm{B}$, White $\mathrm{S}$, et al. Effects of low cardiopulmonary reserve on pancreatic leak following pancreaticoduodenectomy. British Journal of Surgery 2012; 99(9): 1290-1294.

45. Oliak D, Ballantyne GH, Davies RJ, Wasielewski A Schmidt HJ. Short-term results of laparoscopic gastric bypass in patients with $\mathrm{BMI} \geq 60$. Obesity Surgery 2002; 12(5): 643-647 (abstract).
46. Aurora AR, Khaitan L, Saber AA. Sleeve gastrectomy and the risk of leak: A systematic analysis of 4,888 patients, Surgical Endoscopy 2012:1-7 (abstract).

47. Older P, Hall A, Hader R. Cardiopulmonary exercise testing as a screening test for perioperative management of major surgery in the elderly. Chest 1999; 116(2): 355.

48. Whooley BP, Law S, Murthy SC, Alexandrou A, Wong J. Analysis of reduced death and complication rates after esophageal resection. Annals of Surgery $2001 ; 233(3): 338$

49. Low J. Cardiopulmonary exercise testing and survival after major surgery. British Journal of Anaesthesia 2013; 110(3): 484-484.

50. Russ AJ, Weber SM, Rettammel RJ, Mahvi DM, Rikkers LF , Cho CS. Impact of selection bias on the utilization of adjuvant therapy for pancreas adenocarcinoma. Annals of Surgical Oncology 2010; 17(2): 371-376.

51. Mayo SC, Gilson MM, Herman JM, Cameron JL, Nathan $\mathrm{H}$, Edil BH, et al. Management of patients with pancreatic adenocarcinoma: National trends in patient selection, operative management, and use of adjuvant therapy. Journal of the American College of Surgeons 2012; 214(1): 33-45. 